\title{
Undifferentiated embryonal sarcoma of the liver in an adult patient
}

\author{
Kyu Ho Lee', Mussin Nadiar Maratovich², and Kyoung-Bun Lee' \\ 'Department of Pathology, Seoul National University Hospital, Seoul, Korea; ${ }^{2}$ Department of Emergency, City Hospital \#1, Astana,
} Kazakhstan

Undifferentiated embryonal sarcoma of the liver (UESL) is rare primary hepatic sarcoma and is known to occur in pediatric patients. This case is the UESL occurred in a 51-year old male patient. Multilocular cystic lesion was composed of primitive spindle cells without specific differentiation. This rare case would help to review differential diagnosis of primary sarcoma in liver and cystic neoplasm of the liver. (Clin Mol Hepatol 2016;22:292-295)

Keywords: Undifferentiated embryonal sarcoma of the liver; Hepatic sarcoma

\section{INTRODUCTION}

Undifferentiated embryonal sarcoma of the liver (UESL) has also been called undifferentiated sarcoma of the liver or malignant mesenchymoma of the liver. As the disease name and synonyms imply, UESL chiefly occurs in pediatric patients and is composed of poorly differentiated cells of undetermined cellular lineage other than mesenchymal differentiation. Primary malignant mesenchymal tumors in the liver are relatively rare compared with carcinomas originating from hepatocytes or cholangiocytes. Although there are some sarcomas that can primarily occur in the liver, such as angiosarcoma, rhabdomyosarcoma, leiomyosarcoma, and synovial sarcoma, their histologic features and diagnostic criteria are similar to those of soft tissue tumors. UESL is a unique primary sarcoma in liver that has specific histologic and clinical characteristics and has no similar entities among soft tissue tumors. UESL is the third most common malignant tumor in older children, fol-

\section{Abbreviations:}

anti-HCV, anti-hepatitis C viral antibody; HBsAg, hepatitis B surface antigen; $\mathrm{MRI}$, magnetic resonance Imaging; UESL, undifferentiated embryonal sarcoma of the liver lowing hepatoblastoma and hepatocellular carcinoma, and 75\% of cases have been diagnosed in children aged 6-15 years. ${ }^{1,2}$ Here I report a undifferentiated embryonal sarcoma case that occurred in an adult patient and review the relevant literature.

\section{CASE}

A 51-year-old man was admitted for abdominal pain that developed 2 days ago. Abdominal magnetic resonance imaging (MRI) showed a $15 \mathrm{~cm}$ lobulated cystic mass with thin enhancing septa in the right lobe of the liver with fluid levels suggesting internal hemorrhage. Radiologic findings suggested biliary cystadenoma as the differential diagnosis.

Serum levels of tumor markers (carcinoembryonic antigen, CA19-9, alpha-fetoprotein, and prostate specific antigen) were all within normal limits and he was negative for hepatic viral markers 

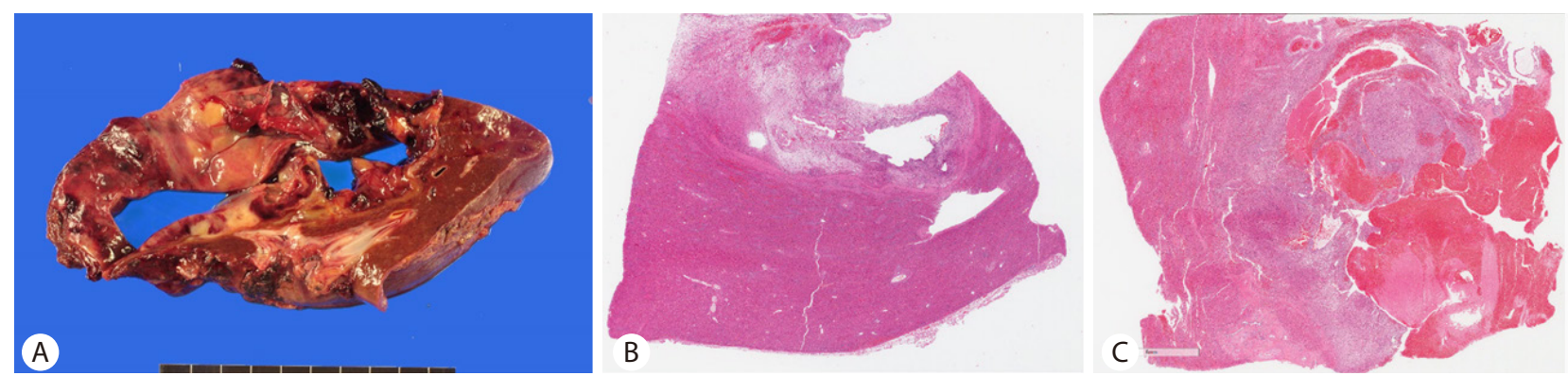

Figure 1. Gross picture and scan view. (A) Multilobular cyst lesion with dark red bloody material on cyst wall. (B) Myxoid stroma of cyst wall. (C) Hypercellular solid mass with hemorrhage [H\&E stain, original magnification $\times 12(B),(C)]$.
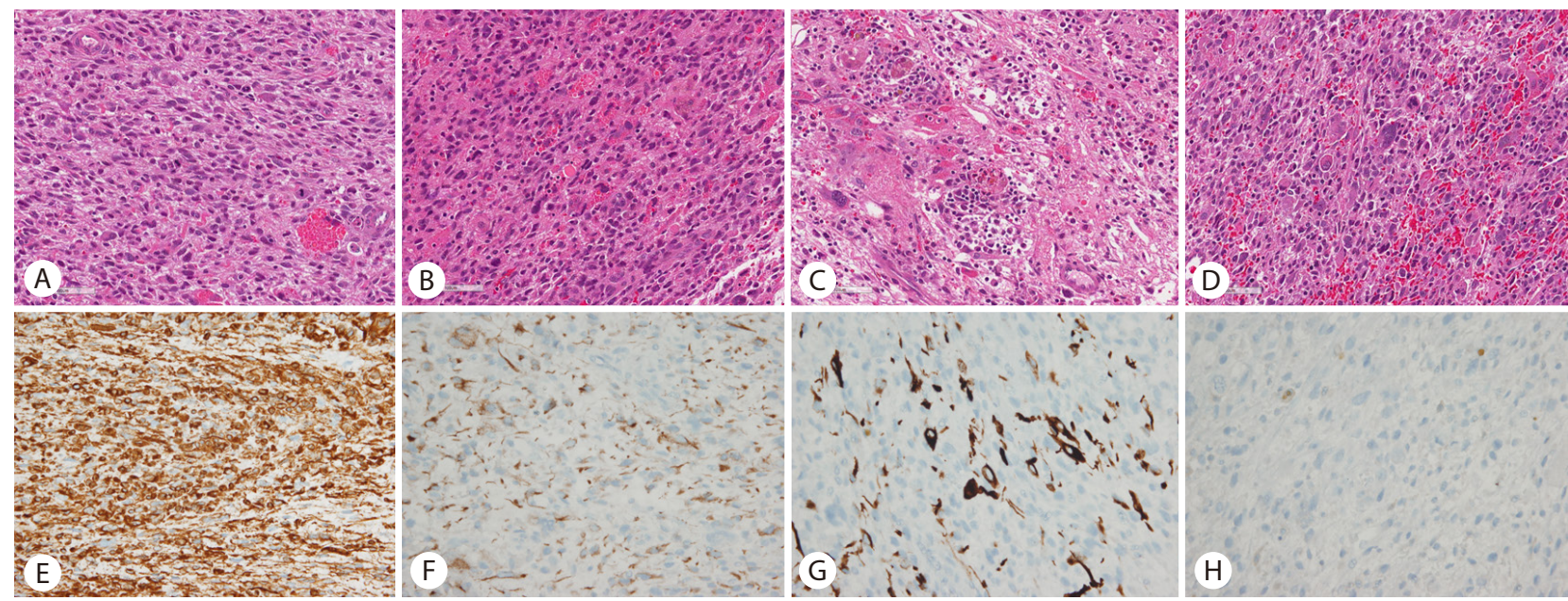

Figure 2. Microscopic and immunohistochemical findings. (A) Plump ovoid or spindle cells with diffuse sheet-like pattern and frequent mitosis. (B) Pinkish hyaline globules or clusters of eosinophilic granules. (C) Intracytoplasmic eosinophilic gradnules, reminiscent of hemophagocytic cells. (D) Multinucleated giant cells [(A)-(D) H\&E stain, original magnification $\times 400]$. (E) Diffuse Positivity for vimentin. (F) Focal positivity for cytokeratin. (G) Focal positivity for desmin. (H) Negative for myogenin. $(E-H)$ immunohistochemistry, original magnification $\times 400$.

(HBsAg and anti-HCV). He had regular follow-up for cardiac valve disease and no history of a previously diagnosed malignancy. Right hemihepatectomy was performed.

\section{PATHOLOGIC FINDINGS}

On gross examination, a $14.0 \times 10.0 \times 5.5 \mathrm{~cm}$ multilocular cystic lesion was identified in non-cirrhotic hepatic parenchyma

The cystic contents were bloody and the cyst wall was covered with dark red bloody material. Thickness of the cyst wall varied from $0.2 \mathrm{~cm}$ to $2.5 \mathrm{~cm}$. The thick cystic wall showed a mixed yellow and white, glistening, myxoid fish-flesh-like cut surface with hemorrhage (Fig. 1A, B).

Under microscopy, the thick cystic wall was a hypercellular solid lesion bordered by non-neoplastic hepatic parenchyma with a thin fibrous capsule (Fig. 1C). The cells were plump ovoid or spindle cells that showed a diffuse sheet-like pattern without an organoid pattern (Fig. 2A) The tumor stroma was loose edematous myxoid or fibrous in the periphery of the lesion. The tumor cells had a moderate amount of cytoplasm and hyperchromatic nuclei with severe pleomorphism. Some multinucleated giant cells were identified (Fig. 2D). Pinkish hyaline globules or clusters of eosinophilic granules were frequently found in the extracellular area or in the intracellular cytoplasm reminiscent of hemophagocytic cells (Fig. $2 B, C)$. Mitosis were frequently found, up to $12 / 10$ high power field (HPF; $\times 400)$ (Fig. 1A). The thin cystic wall had similar histologic features to the solid lesion but the cyst wall had no lining cells. Some glandular structure with cystic changes was entrapped in the periphery of the tumor.

Immunohistochemical staining revealed that the tumor cells were diffusely reactive for vimentin, and focally positive for desmin, smooth muscle actin, and cytokeratin, but not positive for S-100, CD117, myogenin, CD34, or Ets-related gene (ERG) (Fig.2F-H). 


\section{DISCUSSION}

UESL is a rare primary mesenchymal tumor that usually occurs in older children, but some cases have been reported in adults. About 46 articles related with adult USEL from 1977 to 2015 have been published. A solid mass with extensive cystic or necrotic changes is a notable manifestation of UESL. This frequent cystic change in UESL and the rarity of UESL in adults often leads to a misdiagnosis during preoperative evaluation. Commonly reported differential diagnoses are hepatic abscess, hydatid cyst, hemorrhage cystic tumor, and klatskin tumor. ${ }^{3.7}$ In pediatric patients, the main differential diagnosis of UESL before treatment is mesenchymal hamartoma, which is the second most common benign liver tumor after infantile hemangioendothelioma. ${ }^{8}$ Generally, serum tumor markers are within the normal range, but one case of erythropoietin-secreting UESL has been reported. ${ }^{9}$

The underlying histogenesis and etiology of UESL are unknown. Histologically, tumor cells have high pleomorphism, hyperchromasia, and abundant mitoses, representing an undifferentiated and highly proliferative phenotype. Multiple intracellular or extracellular eosinophilic globules that are positive for Periodic acid-Schiff (PAS) stain and resistant to diastase PAS are characteristic histologic features of UESL. Ultrastructurally, these globules are electron-dense lysosomes with dense precipitates and dilated rough endoplasmic reticulum with scanty actin filaments in the cytoplasm, suggesting fibroblastic, fibrohistiocytic, and undifferentiated cells as a cellular lineage..$^{10}$ Immunohistochemically, vimentin is consistently expressed in the tumor cells but desmin, keratin, $\alpha$-smooth muscle actin, $\alpha$-1-antichymotrypsin, CD10, CD68, and calponin show variable expression. ${ }^{11-13}$ Glypican 3, a fetal oncoprotein that is normally expressed during embryogenic development, has been reported to be expressed in UESL, suggesting that UESL has an immature phenotype. ${ }^{14}$

Alterations of TP53; allelic imbalances of 1p, 80, and 20q; and loss of heterozygosity of 7p, 11p, 17p, and 22q have been reported in UESDL. However, beta-catenin activation, a commonly-altered pathway in pediatric tumors, has not been identified. ${ }^{15} \mathrm{~A}$ few cases of UESL arising in mesenchymal hamartomas have been reported in pediatric patients and they showed common cytogenetic alterations, $t(11 ; 19)(q 13 ; q 13.4)$, suggesting a common pathogenic pathway of the two lesions. ${ }^{16}$ The MALAT1 gene was the break-apart site at 11q13, and it has been reported in a subset of renal tumors. The other breakpoint at 19q13.4 was the MHLB1 locus that does not contain a known gene. ${ }^{16}$

Pathologic diagnosis of UESL is an exclusive diagnosis because of the lack of specific diagnostic markers and its primitive histology. Among the malignant pediatric tumors in the liver, embryonal rhabdomyosarcoma and malignant rhabdoid tumor have to be preferentially excluded, since they share histologic features with UESL. Co-expression of desmin, myogenin, and myoglobin suggests rhabdomyosarcoma and loss of expression of INI1 suggests a malignant rhabdoid tumor.

In adult patients, differential points are somewhat different from those of pediatric patients. Because primary sarcoma of liver is rare in adult, metastatic sarcoma should first be excluded and then sarcomatoid carcinoma should be excluded. Because the primary carcinomas in the liver or peritoneum, such as hepatocellular carcinoma, cholangiocarcinoma, and mesothelioma, can be transformed to have both mesenchymal and epithelial differentiation, epithelial differentiation of tumor cells and the co-existence of carcinoma should be excluded to confirm UESL. Because this case also had entrapped bile ducts in the peripheral portion and showed cystic changes, multiple tumor sections were obtained to examine for evidence of concomitant epithelial neoplasms, including adenocarcinoma or dysplasia.

The prognosis of UESL has been poor until recently, but some improvements in survival have been achieved in both pediatric and adult patients by combined modality treatments. ${ }^{17}$ Repeated complete resection, neoadjuvant or adjuvant chemotherapy, and liver transplantation have been applied as multimodal treatment. ${ }^{17}$ The patient in this case received adjuvant chemotherapy after complete resection and has no evidence of disease recurrence to date.

This case presented as a cystic lesion in the liver and had typical histological features of UESL. It is a rare case of a malignant pediatric tumor occurring in an adult patient.

\section{Acknowledgments}

This research was supported by a grant of the Korea Health Technology R\&D Project through the Korea Health Industry Development Institute (KHIDI), funded by the Ministry of Health \& Welfare, Republic of Korea (grant number : HI14C3298).

\section{Conflicts of Interest}

The authors have no conflicts to disclose.

\section{REFERENCES}

1. Stocker JT. An approach to handling pediatric liver tumors. Am J Clin Pathol 1998;109:S67-S72.

2. Stocker JT, Ishak KG. Undifferentiated (embryonal) sarcoma of the 
liver: report of 31 cases. Cancer 1978;42:336-348.

3. Xie ZY, Li LP, Wu WJ, Sun DY, Zhou MH, Zhao YG. Undifferentiated embryonal sarcoma of the liver mistaken for hepatic abscess in an adult. Oncol Lett 2014;8:1184-1186.

4. Yoon JY, Lee JM, Kim do Y, Choi GH, Park YN, Chung JW, et al. A case of embryonal sarcoma of the liver mimicking a hydatid cyst in an adult. Gut Liver 2010;4:245-249.

5. Tsukada A, Ishizaki Y, Nobukawa B, Kawasaki S. Embryonal sarcoma of the liver in an adult mimicking complicated hepatic cyst: MRI findings. J Magn Reson Imaging 2010;31:1477-1480.

6. Kim HH, Kim JC, Park EK, Hur YH, Koh YS, Cho CK, et al. Undifferentiated embryonal sarcoma of the liver presenting as a hemorrhagic cystic tumor in an adult. Hepatobiliary Pancreat Dis Int 2011;10:657660.

7. Lee JA, Kim TW, Min JH, Byon SJ, Jang SH, Choi SY, et al. [A case of undifferentiated (embryonal) liver sarcoma mimicking klatskin tumor in an adult]. Korean J Gastroenterol 2010;55:144-148.

8. Wildhaber BE, Montaruli E, Guérin F, Branchereau S, Martelli $H$, Gauthier $F$. Mesenchymal hamartoma or embryonal sarcoma of the liver in childhood: a difficult diagnosis before complete surgical excision. J Pediatr Surg 2014;49:1372-1377.

9. Lin JM, Heath JE, Twaddell WS, Castellani RJ. Undifferentiated sarcoma of the liver: a case study of an erythropoietin-secreting tumor. Int J Surg Pathol 2014;22:555-558.

10. Agaram NP, Baren A, Antonescu CR. Pediatric and adult hepatic embryonal sarcoma: a comparative ultrastructural study with morphologic correlations. Ultrastruct Pathol 2006;30:403-408.

11. Duenas D, Huanca L, Cordero M, Webb P, Ruiz E. [Undifferentiated (embryonal) liver sarcoma: reviaew of 6 cases in National Cancer In- stitute, Lima, Peru. Review of the literature]. Rev Gastroenterol Peru 2016;36:71-76.

12. Kiani B, Ferrell LD, Qualman S, Frankel WL. Immunohistochemical analysis of embryonal sarcoma of the liver. Appl Immunohistochem Mol Morphol 2006;14:193-197.

13. Pérez-Gómez RM, Soria-Céspedes D, de León-Bojorge B, Ortiz-Hidalgo C. Diffuse membranous immunoreactivity of CD56 and paranuclear dot-like staining pattern of cytokeratins AE1/3, CAM5.2, and OSCAR in undifferentiated (embryonal) sarcoma of the liver. Appl Immunohistochem Mol Morphol 2010;18:195-198.

14. Kinoshita Y, Tanaka S, Souzaki R, Miyoshi K, Kohashi K, Oda Y, et al. Glypican 3 expression in pediatric malignant solid tumors. Eur $J$ Pediatr Surg 2015;25:138-144.

15. Lepreux S, Rebouissou S, Le Bail B, Saric J, Balabaud C, Bloch $B$, et al. Mutation of TP53 gene is involved in carcinogenesis of hepatic undifferentiated (embryonal) sarcoma of the adult, in contrast with Wnt or telomerase pathways: an immunohistochemical study of three cases with genomic relation in two cases. J Hepatol 2005;42:424-429.

16. Rajaram V, Knezevich S, Bove KE, Perry A, Pfeifer JD DNA sequence of the translocation breakpoints in undifferentiated embryonal sarcoma arising in mesenchymal hamartoma of the liver harboring the $t(11 ; 19)(q 11 ; q 13.4)$ translocation. Genes Chromosomes Cancer 2007:46:508-513.

17. Techavichit P, Masand PM, Himes RW, Abbas R, Goss JA, Vasudevan $S A$, et al. Undifferentiated embryonal sarcoma of the liver (UESL): a single-center experience and review of the literature. J Pediatr Hematol Oncol 2016;38:261-268. 